\title{
Bianchi Type-V Cosmology with Magnetized Anisotropic Dark Energy
}

\begin{tabular}{|r|l|}
\hline Journal: & Canadian Journal of Physics \\
\hline Manuscript ID & cjp-2016-0777.R1 \\
\hline Manuscript Type: & Article \\
\hline Date Submitted by the Author: & $29-$ Nov-2016 \\
\hline Complete List of Authors: & $\begin{array}{l}\text { Shamir, M. Farasat; National University of Computer \& Emerging Sciences, } \\
\text { Sciences \& Humanities } \\
\text { Ali, Asad; National University of Computer and Emerging Sciences }\end{array}$ \\
\hline Keyword: & Anisotropy, Dark Energy, Exact Solutions, Bianchi V, Cosmology \\
\hline \multicolumn{2}{|c}{} \\
\hline
\end{tabular}

SCHOLARONE ${ }^{\text {IM }}$

Manuscripts 


\title{
Bianchi Type- $V$ Cosmology with Magnetized Anisotropic Dark Energy
}

\author{
M. Farasat Shamir*and Asad Ali ${ }^{\dagger}$ \\ Department of Sciences and Humanities, \\ National University of Computer and Emerging Sciences, \\ Lahore Campus, Pakistan.
}

\begin{abstract}
We study anisotropic universe in the presence of magnetized dark energy. Bianchi type- $V$ cosmological model is considered for this purpose. The energy-momentum tensor consists of anisotropic fluid with uniform magnetic field of energy density $\rho_{B}$. Exact solutions to the field equations are obtained without using conventional assumptions like constant deceleration parameter. In particular, a general solution is obtained which further provides different classes of solutions. Only three cases have been discussed for the present analysis, i.e. linear, quadratic and exponential. Graphical analysis of the solutions is done for all the three classes. The behavior of the model using some important physical parameters is discussed in the presence of magnetic field.
\end{abstract}

Keywords: Dark Energy; Anisotropy; Exact Solutions.

PACS: : 04.20.Jb; 98.80.-k; 98.80.Jk.

\section{Introduction}

In last decade researchers (Supernova Cosmology Projects), provided some evidence that expansion of the universe was accelerating [1]-[4]. The universe

\footnotetext{
*farasat.shamir@nu.edu.pk

†asadali.nu@gmail.com
} 
consists of 70 percent of the dark energy which is a mysterious substance, but there is no clear explanation of it. Dark energy has been traditionally characterized by equation of state $(\mathrm{EoS})$ parameter $\omega=\frac{p}{\rho}$, which is not necessarily a constant. The simplest dark energy case is the vacuum energy, which is mathematically equivalent to cosmological constant. The other possibilities are quintessence, phantom energy and quintom which have time dependent EoS parameter. Also the results coming from SN Ia data collaborated with CMBR anisotropy and galaxy clustering statistics suggest $-1.67<\omega<-0.62$ $[5,6]$. There are various models available in literature that have been proposed to explain dark energy, for details see [7]-[13]. However, it is not at all mandatory to use a constant value of $\omega$.

The special law of variation of Hubble parameter in Friedman Robertson Walker (FRW) space-time was proposed by Berman [14], which gave a constant value of deceleration parameter. Such a law of variation for Hubble parameter is consistent with the observations and is also approximately valid for slowly time varying models. Laws governing the universe's scale provide an explicit form of FRW universe model and facilitate to describe accelerating as well as decelerating models of evolution of the universe. Recently, anisotropic Bianchi type- $I$ cosmological models have been investigated in the context of $f(R)$ and $f(G)$ modified theories of gravity [15]-[17].

Pradhan and Saha [18] constructed locally rotationally symmetric (LRS) Bianchi type- $I$ cosmological models with dynamically anisotropic dark energy and perfect fluid. Akarsu and Kilinc [19] also investigated LRS Bianchi typeI cosmology with anisotropic dark energy and they concluded that the dark energy is slightly interacting, had dynamical energy density and anisotropic EoS parameter. The same authors [20] found the exact solutions of Bianchi type- III models with anisotropic dark energy. Yadav [21] examined Bianchi type- III anisotropic dark energy models with constant deceleration parameter. Bianchi type-III magnetized anisotropic dark energy models with constant deceleration parameter and dark energy models with anisotropic fluid in Bianchi type- $V I_{0}$ with time dependent deceleration parameter have been investigated by Tade et al. [22] and Pradhan et al. [23] respectively. Saha [24] has studied the evolution of the universe filled with dark energy within the scope of a Bianchi type- $V$ model.

Using the proportionality condition and variational law of Hubble parameter, the exact solutions of field equations are explored by many researchers. Priyanka et al. [25] gave the exact solutions of Einstein's field equations for a Bianchi type- $V I_{0}$ space-time filled with perfect fluid satisfying the barotropic 
EoS under the supposition that the expansion scalar was proportional to shear scalar. Saha [26] studied Bianchi type-VI dark energy model with varying EoS parameter. Sharif and Shamir [27] did work on exact solutions of Bianchi types $I$ and $V$ space-time in $f(R)$ theory of gravity. The same authors [28] also studied the non-vacuum solutions of these models. Dynamics of Bianchi type- $I$ universe with magnetized anisotropic dark energy has been discussed by Sharif and Zubair [29]. They concluded that the universe model as well as anisotropic fluid did not approach isotropy through the evolution of the universe. The same authors [30] studied the effects of electromagnetic field on the dynamics of Bianchi type $V I_{0}$ universe with anisotropic dark energy. Thus, it seems interesting to explore further about the universe with anisotropic dark energy.

In this paper, we are focussed to explore Bianchi Type- $V$ cosmology with magnetized anistropic dark energy. It is mentioned hare that the exact solutions to the field equations are obtained without using conventional assumptions like constant deceleration parameter. In particular, a general solution is obtained which further provides different classes of solutions. Only three classes have been discussed for the present analysis, i.e. linear, quadratic and exponential. Graphical analysis of the solutions for the discussed classes is given. The plan of paper is as follows: In section 2, we give Bianchi type- $V$ field equations and energy conservation equation. Section $\mathbf{3}$ provides qualitative analysis of some exact solutions along with discussion of some important physical parameters. Summary and concluding remarks are given in the last section.

\section{Einstein Field Equations and Exact Solu- tions}

The line element for Bianchi type- $V$ space-time is given as [27]

$$
d s^{2}=d t^{2}-a^{2} d x^{2}-b^{2} e^{2 m x} d y^{2}-c^{2} e^{2 m x} d z^{2}
$$

where the scale factors $a$ and $b$ and $c$ are functions of cosmic time $t$ only. Here we take energy-momentum tensor for the magnetized anisotropic DE 
fluid in the following form

$$
T_{b}^{a}=\left[\begin{array}{cccc}
\rho+\rho_{B} & 0 & 0 & 0 \\
0 & \rho_{B}-\omega \rho & 0 & 0 \\
0 & 0 & -(\omega+\delta) \rho-\rho_{B} & 0 \\
0 & 0 & 0 & -(\omega+\gamma) \rho-\rho_{B}
\end{array}\right]
$$

where $\rho$ is the energy density of the fluid, $\rho_{B}$ magnetic energy density, $\omega$ deviation free EoS parameter of the fluid and $\delta, \gamma$ shows the deviation from $\omega$ along $y$ and $z$ axis respectively which are called skewness parameters. Using Eq.(1), with gravitational units, the Einstein field equations take the form

$$
\begin{gathered}
\frac{a^{\prime} b^{\prime}}{a b}+\frac{b^{\prime} c^{\prime}}{b c}+\frac{a^{\prime} c^{\prime}}{a c}-\frac{3 m^{2}}{a^{2}}=\left(\rho_{B}+\rho\right), \\
{\left[\frac{b^{\prime \prime}}{b}+\frac{c^{\prime \prime}}{c}+\frac{b^{\prime} c^{\prime}}{b c}-\frac{m^{2}}{a^{2}}\right]=\left(\rho_{B}-\omega \rho\right),} \\
\left.\left[\frac{a^{\prime \prime}}{a}+\frac{c^{\prime \prime}}{c}+\frac{a^{\prime} c^{\prime}}{a c}-\frac{m^{2}}{a^{2}}\right]=-\left[(\omega+\delta) \rho+\rho_{B}\right)\right], \\
\left.\left[\frac{a^{\prime \prime}}{a}+\frac{b^{\prime \prime}}{b}+\frac{a^{\prime} b^{\prime}}{a b}-\frac{m^{2}}{a^{2}}\right]=-\left[(\omega+\gamma) \rho+\rho_{B}\right)\right], \\
{\left[\frac{2 a^{\prime}}{a}-\frac{b^{\prime}}{b}-\frac{c^{\prime}}{c}\right]=0 .}
\end{gathered}
$$

These are five non-linear differential equations with eight unknowns namely $a, b, c, \rho, \rho_{B}, \gamma, \delta$, and $\omega$. Therefore, we need some additional constraints in order to find the solutions. From Eq.(6), we get

$$
a^{2}=b c
$$

Energy conservation equation $T_{\nu ; \mu}^{\mu}=0$ provides

$$
\rho^{\prime}+\rho\left(\frac{b^{\prime}}{b} \delta+\gamma \frac{c^{\prime}}{c}\right)+(\rho+\omega \rho)\left[\frac{a^{\prime}}{a}+\frac{b^{\prime}}{b}+\frac{c^{\prime}}{c}\right]+\rho_{B}^{\prime}+2 \rho_{B}\left(\frac{b^{\prime}}{b}+\frac{c^{\prime}}{c}\right)=0
$$

This equation contains magnetic and non-magnetic part. Assuming magnetic and non-magnetic part zero and solving its magnetic part, we get

$$
\rho_{B}=\frac{c_{1}}{(b c)^{2}}
$$


where $c_{1}$ is an integration constant. The filed equations are highly nonlinear and it seems difficult to solve them with eight unknowns. So to solve the field equations, we use a physical condition that expansion scalar $\theta$ is proportional to shear scalar $\sigma$ which provides $b=c^{n}$, where $n$ is an arbitrary non-zero real number. We further consider $c$ in a general power law form, i.e., $c=$ $(f(t)+\beta)^{k}$, where $f(t)$ is an arbitrary function of $t$ and $k$ is a non-zero real number. Using these assumptions and Eqs.(2)-(6), we obtain

$$
\begin{aligned}
\rho_{B}=\frac{c_{1}}{(f(t)+\beta)^{2 k(n+1)}}, & (10) \\
\rho=( & \left.\frac{k f(t)^{\prime}}{f(t)+\beta}\right)^{2}\left(\frac{n^{2}+4 n+1}{2}\right)-3\left[\frac{m^{2}}{(f(t)+\beta)^{k(n+1)}}\right]-\frac{c_{1}}{(f(t)+\beta)^{2 k(n+1)}},(11) \\
\omega= & -\frac{2}{\left(f(t)^{\prime}\right)^{2}\left(n^{2}+4 n+1\right)(f(t)+\beta)^{2 k n+2 k}-6 m^{2}(f(t)+\beta)^{k n+k-2}-2 c_{1}(f(t)+\beta)^{2}} \times \\
& {\left[f(t)^{\prime 2} k\left(k n^{2}+1\right)(f(t)+\beta)^{2 k n+2 k}+f(t)^{\prime \prime} k(n+1)(f(t)+\beta)^{2 k n+2 k+1}+\right.} \\
& \left.\left(k f(t)^{\prime}\right)^{2}(f(t)+\beta)^{2 k n+2 k}-m^{2}(f(t)+\beta)^{k n+k+2}-c_{1}(f(t)+\beta)^{2}\right], \\
\delta= & -\frac{1}{\left(k f(t)^{\prime}\right)^{2}\left(n^{2}+4 n+1\right)-6 m^{2}(f(t)+\beta)^{2-k n-k}-2 c_{1}(f(t)+\beta)^{2}}\left[f(t)^{\prime 2} k(n+1)\right. \\
& -2 k(n+1)-2(f(t)+\beta) f(t)^{\prime \prime} k(n+1)-4\left(f(t)^{\prime}\right)^{2} k n(k n-1)-4 f(t)^{\prime \prime 2} k n(f(t)+\beta) \\
& \left.+2\left(k f(t)^{\prime}\right)^{2}(n+1)-4 n\left(k f(t)^{\prime}\right)^{2}+8 c_{1}(f(t)+\beta)^{2-2 k n-2 k}\right], \\
\gamma= & -\frac{1}{\left(k f(t)^{\prime}\right)^{2}\left(n^{2}+4 n+1\right)(f(t)+\beta)^{2 k n+2 k}-6 m^{2}(f(t)+\beta)^{2-k n-k}-2 c_{1}(f(t)+\beta)^{2} \times} \\
& {\left[f(t)^{\prime 2} k(n+1)^{2}(f(t)+\beta)^{2 k n+2 k}-2 k(n+1)(f(t)+\beta)^{2 k n+2 k}+2\left(f(t)^{\prime \prime}\right) k(n+1) \times\right.} \\
& (f(t)+\beta)^{2 k n+2 k+1}-4 k(k-1)(f(t)+\beta)^{2 k n+2 k+1}-4 f(t)^{\prime \prime}(f(t)+\beta)^{2 k n+2 k+2} \\
+ & \left.2 n(n+1)\left(f(t)^{\prime} k\right)^{2}(f(t)+\beta)^{2 k n+k}-4 n(f(t)+\beta)^{2 k n+2 k}\left(k f(t)^{\prime}\right)^{2}+8 c_{1}(f(t)+\beta)^{2}\right] .
\end{aligned}
$$

So far we have found the values of the unknowns $\rho, \rho_{B}, \gamma, \delta$ and $\omega$ in terms of unknown $f(t)$. 


\section{Different Cases of Some Exact Solutions}

In this section, we will investigate different possibilities of solutions along with the graphical analysis. Polynomial form of $f(t)$ are considered and only linear and quadratic cases are analyzed. The solutions for exponential $f(t)$ are also discussed.

\subsection{Linear case}

When $f(t)=\xi t$, where $\xi$ is an arbitrary non-zero constant, the solution metric takes the form

$$
d s^{2}=d t^{2}-(\xi t+\beta)^{k n+k} d x^{2}-(\xi t+\beta)^{2 k n} e^{2 m x} d y^{2}-(\xi t+\beta)^{2 k} e^{2 m x} d z^{2}
$$

while the expressions for energy density and magnetic energy density takes the form

$$
\begin{gathered}
\rho=\left(\frac{k \xi}{\xi t+\beta}\right)^{2}\left(\frac{n^{2}+4 n+1}{2}\right)-3\left[\frac{m^{2}}{(\xi t+\beta)^{k(n+1)}}\right]-\frac{c_{1}}{(\xi t+\beta)^{2 k(n+1)}}, \\
\rho_{B}=\frac{c_{1}}{(\xi t+\beta)^{2 k(n+1)}} .
\end{gathered}
$$

Its graph is shown in figure $1(1 \mathrm{a}, 1 \mathrm{~b})$. The graph shows that energy density of magnetic field increases with the increase of $t$ and $n$, while energy density of the fluid decreases with the increase of $t$ and $n$. This indicates the magnetic dominated universe as the time grows. The expressions for other parameters $\omega, \delta$ and $\gamma$ take the form

$$
\omega=\frac{-2\left[\xi^{2} k\left(k n^{2}+1\right)(\xi t+\beta)^{2 k n+2 k}+(k \xi)^{2}(\xi t+\beta)^{2 k n+2 k}-m^{2}(\xi t+\beta)^{k n+k+2}-c_{1}(\xi t+\beta)^{2}\right]}{(\xi k)^{2}\left(n^{2}+4 n+1\right)(\xi t+\beta)^{2 k n+2 k}-6 m^{2}(\xi t+\beta)^{k n+k-2}-2 c_{1}(\xi t+\beta)^{2}},
$$

$$
\begin{aligned}
\delta= & -\frac{1}{(k \xi)^{2}\left(n^{2}+4 n+1\right)-6 m^{2}(\xi t+\beta)^{2-k n-k}-2 c_{1}(\xi t+\beta)^{2}}\left[\xi^{2} k(n+1)-2 k(n+1)\right. \\
& \left.-4(\xi)^{2} k n(k n-1)+2(k \xi)^{2}(n+1)-4 n(k \xi)^{2}+8 c_{1}(\xi+\beta)^{2-2 k n-2 k}\right]
\end{aligned}
$$




$$
\begin{aligned}
\gamma= & -\frac{1}{\left(k f(t)^{\prime}\right)^{2}\left(n^{2}+4 n+1\right)(f(t)+\beta)^{2 k n+2 k}-6 m^{2}(f(t)+\beta)^{2-k n-k}-2 c_{1}(f(t)+\beta)^{2}} \times \\
& {\left[\xi^{2} k(n+1)^{2}(\xi t+\beta)^{2 k n+2 k}-2 k(n+1)(\xi t+\beta)^{2 k n+2 k}-4 k(k-1)(\xi t+\beta)^{2 k n+2 k+1}\right.} \\
& \left.+2 n(n+1)(\xi k)^{2}(\xi t+\beta)^{2 k n+k}-4 n(\xi t+\beta)^{2 k n+2 k}(k \xi)^{2}+8 c_{1}(\xi t+\beta)^{2}\right] .
\end{aligned}
$$

The graphical behavior of $\omega, \delta$ and $\gamma$ is shown in figures 2 and 3 . It can be seen from graph that for a certain value of anisotropic parameter $n$ and $t, \omega=-1$. This is interesting as it has been proved that the expansion of the universe is accelerating when $\omega \approx-1$ [31]. Moreover, the phantom like dark energy is found to be in the region where $\omega<-1$ and the universe with phantom dark energy ends up with a finite time future singularity known as cosmic doomsday or big rip [32].

\subsection{Quadratic case}

For $f(t)=\xi t^{2}+\zeta t+\lambda$ where $\xi, \lambda, \zeta$ are arbitrary non-zero constants, the solution metric becomes

$d s^{2}=d t^{2}-\left(\xi t^{2}+\zeta t+\lambda+\beta\right)^{k n+k} d x^{2}-\left(\xi t^{2}+\zeta t+\lambda+\beta\right)^{2 k n} e^{2 m x} d y^{2}-\left(\xi t^{2}+\zeta t+\lambda+\beta\right)^{2 k} e^{2 m x} d z^{2}$.

In this case $\rho, \rho_{B}, \omega, \delta$ and $\gamma$ turn out to be

$$
\begin{gathered}
\rho=\left(\frac{k(2 \xi t+\zeta)}{\xi t^{2}+\zeta t+\lambda+\beta}\right)^{2}\left(\frac{n^{2}+4 n+1}{2}\right)-\frac{3 m^{2}}{\left(\xi t^{2}+\zeta t+\lambda+\beta\right)^{k(n+1)}}-\frac{c_{1}}{\left(\xi t^{2}+\zeta t+\lambda+\beta\right)^{2 k(n+1)}} \\
\rho_{B}=\frac{c_{1}}{\left(\xi t^{2}+\zeta t+\lambda+\beta\right)^{2 k(n+1)}}, \\
\omega=-2\left[(2 \xi t+\zeta t)^{2} k^{2}\left(n^{2}+4 n+1\right)\left(\xi t^{2}+\zeta t+\lambda+\beta\right)^{2 k n+2 k}-6 m^{2}\left(\xi t^{2}+\zeta t+\lambda+\beta\right)^{k n+k-2}\right. \\
\left.-2 c_{1}\left(\xi t^{2}+\zeta t+\lambda+\beta\right)^{2}\right]^{-1}\left[4 k \xi^{2} t^{2}\left(k n^{2}+1\right)\left(\xi t^{2}+\zeta t+\lambda+\beta\right)^{2 k n+2 k}+2 k \xi(n+1) \times\right. \\
\left.\left(\xi t^{2}+\zeta t+\lambda+\beta\right)^{2 k n+2 k}-m^{2}\left(\xi t^{2}+\zeta t+\lambda+\beta\right)^{k n+k+2}-c_{1}\left(\xi t^{2}+\zeta t+\lambda+\beta\right)^{2}\right], \quad(24)
\end{gathered}
$$




$$
\begin{aligned}
\delta= & -\frac{1}{k^{2}(\xi t+\zeta)^{2}\left(n^{2}+4 n+1\right)-6 m^{2}\left(\xi t^{2}+\zeta t+\lambda+\beta\right)^{2-k n-k}-2 c_{1}\left(\xi t^{2}+\zeta t+\lambda t+\beta\right)^{2}} \times \\
& {\left[(\xi t+\zeta)^{2} k(n+1)-2 k(n+1)-4 k \xi(n+1)\left(\xi t^{2}+\zeta t+\lambda+\beta\right)-4 k n(k n-1)(2 \xi t+\zeta t)^{2}\right.} \\
& -8 k n\left(\xi t^{2}+\zeta t+\lambda+\beta\right)+2(k(\xi t+\zeta))^{2}(n+1)-4 n(k(\xi t+\zeta))^{2} \\
& \left.+8 c_{1}\left(\xi t^{2}+\zeta t+\lambda+\beta\right)^{2-2 k n-2 k}\right] \\
\gamma= & -\left[k^{2}(2 t \xi+\zeta)^{2}\left(n^{2}+4 n+1\right)\left(t^{2} \xi+t \zeta+\lambda+\beta\right)^{2 k n+2 k}-6 m^{2}\left(t^{2} \xi+\zeta t+\lambda+\beta\right)^{2-k n-k}\right. \\
& \left.-2 c_{1}\left(t^{2} \xi+t \zeta+\lambda+\beta\right)^{2}\right]^{-1}\left[(2 t \xi+\zeta)^{2} k(n+1)^{2}\left(t^{2} \xi+\zeta t+\lambda+\beta\right)^{2 k n+2 k}-2 k(n+1) \times\right. \\
& \left(t^{2} \xi+\zeta t+\lambda+\beta\right)^{2 k n+2 k}+4 k \xi(n+1)\left(t^{2} \xi+t \zeta+\lambda+\beta\right)^{2 k n+2 k+1}-4 k(k-1) \times \\
& \left(t^{2} \xi+t^{2} \zeta+\lambda+\beta\right)^{2 k n+2 k+1}-8 \xi\left(t^{2} \xi+t \zeta+\lambda+\beta\right)^{2 k n+2 k+2}+2 n(n+1)((2 t \xi+\zeta) k)^{2} \times \\
& \left.\left(t^{2} \xi+t \zeta+\lambda+\beta\right)^{2 k n+k}-4(2 t \xi+\zeta) k^{2}+8 c_{1}\left(t^{2} \xi+\zeta t+\lambda+\beta\right)^{2}\right]
\end{aligned}
$$

The graphical behavior of all above quantities is similar as compared to the linear case (see figures 4, 5 and 6 ). Thus it is conjectured that by increasing the degree of polynomial, the resulting solutions will have same physical behavior. Now we discuss the exponential case.

\subsection{Exponential case}

Exponential form of $f(t)$, i.e., $f(t)=\psi e^{t}$, where $\psi$ is an arbitrary non-zero real number, yields the solution metric

$d s^{2}=d t^{2}-\left(\psi e^{t}+\beta\right)^{k(n+1)} d x^{2}-\left(\psi e^{t}+\beta\right)^{2 k n} e^{2 m x} d y^{2}-\left(\psi e^{t}+\beta\right)^{2 k} e^{2 m x} d z^{2}$

Here the expressions for the energy density and magnetic energy density turn out to be

$$
\begin{gathered}
\rho=\left(\frac{k\left(\psi e^{t}\right)}{\psi e^{t}+\beta}\right)^{2}\left(\frac{n^{2}+4 n+1}{2}\right)-3\left[\frac{m^{2}}{\left(\psi t^{2}+\psi e^{t}+\beta\right)^{k(n+1)}}\right]-\frac{c_{1}}{\left(\psi e^{t}+\beta\right)^{2 k(n+1)}}, \\
\rho_{B}={\frac{c_{1}}{\left(\psi e^{t}+\beta\right)}}^{2 k(n+1)} .
\end{gathered}
$$


Figure (7) shows that $\rho_{B}$ decreases gradually with the increase of $t$ and $n$ and by setting $t$ and $n$ sufficiently large $\rho_{B} \rightarrow 0$. Energy density of the universe $\rho$ increases gradually as the time grows. The other parameters for this case are as follows:

$$
\begin{aligned}
\omega= & -2\left[k^{2}\left(\psi e^{t}\right)^{2}\left(n^{2}+4 n+1\right)\left(\psi e^{t}+\beta\right)^{2 k n+2 k}-6 m^{2}\left(\psi e^{t}+\beta\right)^{k n+k-2}-2 c_{1}\left(\psi e^{t}+\beta\right)^{2}\right. \\
& ]^{-1}\left[\left(\psi e^{t}\right)^{2} k\left(k n^{2}+1\right)\left(\psi e^{t}+\beta\right)^{2 k n+2 k}+\left(\psi e^{t}\right)^{2} k(n+1)\left(\psi e^{t}+\beta\right)^{2 k n+2 k}+\right. \\
& \left.\left(\psi e^{t} k\right)^{2}\left(\psi e^{t}+\beta\right)^{2 k n+2 k}-m^{2}\left(\psi e^{t}+\beta\right)^{k n+k+2}-c_{1}\left(\psi e^{t}+\beta\right)^{2}\right] \\
\delta= & -\frac{1}{\left(k\left(\psi e^{t}\right)\right)^{2}\left(n^{2}+4 n+1\right)-6 m^{2}\left(\psi e^{t}+\beta\right)^{2-k n-k}-2 c_{1}\left(\psi e^{t}+\beta\right)^{2}}\left[\left(\psi e^{t}\right)^{2} k(n+1)\right. \\
& -2 k(n+1)-2 k\left(\psi e^{t}\right)(n+1)\left(\psi e^{t}+\beta\right)-4 k n(k n-1)\left(\psi e^{t}\right)^{2}- \\
& \left.4 k n\left(\psi e^{t}+\beta\right)+\left(k\left(\psi e^{t}\right)\right)^{2}(n+1)-4 n\left(k\left(\psi e^{t}\right)\right)^{2}+8 c_{1}\left(\psi e^{t}+\beta\right)^{2-2 k n-2 k}\right] \\
\gamma= & -\frac{(31)}{\left(k\left(\psi e^{t}\right)\right)^{2}\left(n^{2}+4 n+1\right)\left(\psi e^{t}+\beta\right)^{2 k n+2 k}-6 m^{2}\left(\psi e^{t}+\beta\right)^{2-k n-k}-2 c_{1}\left(\psi e^{t}+\beta\right)^{2} \times} \\
& {\left[\left(\psi e^{t}\right)^{2} k(n+1)^{2}\left(\psi e^{t}+\beta\right)^{2 k n+2 k}-2 k(n+1)\left(\psi e^{t}+\beta\right)^{2 k n+2 k}+2 k \psi e^{t}(n+1) \times\right.} \\
& \left(\psi e^{t}+\beta\right)^{2 k n+2 k+1}-4 k(n+1)\left(\psi e^{t}+\beta\right)^{2 k n+2 k+1}-4 \psi e^{t}\left(\psi e^{t}+\beta\right)^{2 k n+2 k+1}+ \\
2 n(n+1)\left(\psi e^{t} k\right)^{2}\left(\psi e^{t}+\beta\right)^{2 k n+2 k}+2 n(n+1)((2 \psi t+\zeta) k)^{2}\left(\psi t^{2}+\zeta t+\lambda+\beta\right)^{2 k n+k} & (32) \\
& \left.-4 n\left(\psi e^{t}\right) k^{2}\left(\psi e^{t}+\beta\right)^{2 k n+2 k}+8 c_{1}\left(\psi e^{t}+\beta\right)^{2}\right] .
\end{aligned}
$$

The graphical behavior of $\omega, \delta$ and $\gamma$ is given in figures (8) and (9). It can be seen from graph that $\omega$ possesses negative values justifying expansion of universe and existance of the phantom like dark energy. It is interesting that skewness parameter $\delta$ attains both positive and negative values depending upon $n$. However, $\gamma$ approaches to 0 with the increase in time. Now we discuss some important cosmological parameters in the context of exact solutions. 


\subsection{Some Important Cosmological Parameters}

The volume scale factor $V$ for Bianchi type $V$ spacetime is defined as:

$$
V=a b c e^{2 m x} \text {. }
$$

For linear, quadratic and exponential case, the Volume scale factor turn out to be

$$
\begin{gathered}
V=(\xi t+\beta)^{\frac{3}{2} k(n+1)} e^{2 m x}, \\
V=\left(\xi t^{2}+\zeta t+\lambda+\beta\right)^{\frac{3}{2} k(n+1)} e^{2 m x}, \\
V=\left(\psi e^{t}+\beta\right)^{\frac{3}{2} k(n+1)} e^{2 m x} .
\end{gathered}
$$

The average scale factor $A$ is defined as:

$$
A=\left(a b c e^{2 m x}\right)^{\frac{1}{3}} .
$$

The expressions for average scale factor for linear, quadratic and exponential case turn out to be

$$
\begin{gathered}
A=(\xi t+\beta)^{\frac{k(n+1)}{2}} e^{\frac{2 m x}{3}} \\
A=\left(\xi t^{2}+\zeta t+\lambda+\beta\right)^{\frac{k(n+1)}{2}} e^{\frac{2 m x}{3}} \\
A=\left(\psi e^{t}+\beta\right)^{\frac{k(n+1)}{2}} e^{\frac{2 m x}{3}} .
\end{gathered}
$$

The Average Hubble Parameter $H$ is defined as:

$$
H=\frac{H_{1}+H_{2}+H_{3}}{3} .
$$

where $H_{1}=\frac{a^{\prime}}{a}, H_{2}=\frac{b^{\prime}}{b}$ and $H_{3}=\frac{c^{\prime}}{c}$ are directional Hubble parameters. For linear, quadratic and exponential case, the average Hubble parameter $H$ takes the form

$$
\begin{gathered}
H=\frac{\xi t k(n+1)}{2(\xi t+\beta)}, \\
H=\frac{k(n+1)(2 \xi t+\zeta)}{2\left(\xi t^{2}+\zeta t+\lambda+\beta\right)}, \\
H=\frac{\psi e^{t} k(n+1)}{2\left(\psi e^{t}+\beta\right)} .
\end{gathered}
$$

The expansion scalar $\theta$ and shear scalar $\sigma$ are given by

$$
\theta=\left(\frac{a^{\prime}}{a}+\frac{b^{\prime}}{b}+\frac{c^{\prime}}{c}\right),
$$




$$
\sigma^{2}=\frac{1}{2}\left[\left(\frac{a^{\prime}}{a}\right)^{2}+\left(\frac{b^{\prime}}{b}\right)^{2}+\left(\frac{c^{\prime}}{c}\right)^{2}\right]-\left(\frac{\theta^{2}}{6}\right)
$$

respectively. For linear, quadratic and exponential case, the expansion and shear scalar become

$$
\begin{gathered}
\theta=\frac{3 k(n+1) \xi}{2(\xi t+\beta)}, \quad \sigma^{2}=\left(\frac{\xi k}{\xi t+\beta}\right)^{2}\left(\frac{n^{2}-2 n+1}{4}\right), \\
\theta=\frac{3 k(n+1)(2 \xi t+\zeta)}{2\left(\xi t^{2}+\zeta t+\lambda+\beta\right)}, \quad \sigma^{2}=\left(\frac{2(\xi t+\zeta) k}{\xi t^{2}+\zeta t+\lambda+\beta}\right)^{2}\left(\frac{n^{2}-2 n+1}{4}\right), \\
\theta=\frac{3 \psi e^{t} k(n+1)}{2\left(\psi e^{t}+\beta\right)}, \quad \sigma^{2}=\left(\frac{\psi e^{t} k}{\psi e^{t}+\beta}\right)^{2}\left(\frac{n^{2}-2 n+1}{4}\right) .
\end{gathered}
$$

The deceleration, jerk and snap parameters are defined as:

$$
q=-\frac{1}{H^{2}} \frac{A^{\prime \prime}}{A}, \quad j=-\frac{1}{H^{3}} \frac{A^{\prime \prime \prime}}{A}, \quad s=-\frac{1}{H^{4}} \frac{A^{\prime \prime \prime \prime}}{A} .
$$

The value of these parameters for our solutions turn out to be

$$
\begin{aligned}
& q=-\left(\frac{k(n+1)-1}{k(n+1)}\right), \quad j=-\left(\frac{(k n+k-2)(k n+k-4)}{k^{2}(n+1)^{2}}\right), \\
& s=-\left(\frac{(k n+k-2)(k n+k-4)(k n+k-6)}{k^{3}(n+1)^{3}}\right) .
\end{aligned}
$$

\section{Summary and Conclusion}

This paper is devoted to exploring the exact solutions for spatially homogeneous and anisotropic Bianchi type- $V$ cosmological model with magnetized anisotropic DE fluid having anisotropic EoS. The parameters $\rho, \rho_{B}, \gamma, \delta$ and $\omega$ are obtained by assuming that conservation equation of DE consists of two separate conserved parts i.e. magnetic part and anisotropic part.

We have discussed some physical aspects of the model in the presence of magnetic field. Assuming magnetic part to be zero, we found $\rho_{B}$. We have considered only three cases, linear, quadratic, and exponential for the present study. It is conjectured that the solutions for cubic case and higher degree polynomials in general will have same physical behavior as that of linear and quadratic cases. We have calculated the unknowns $\rho, \rho_{B}, \gamma, \delta$ and $\omega$ and their graphical analysis is given. It is worth mentioning here that we have not used 

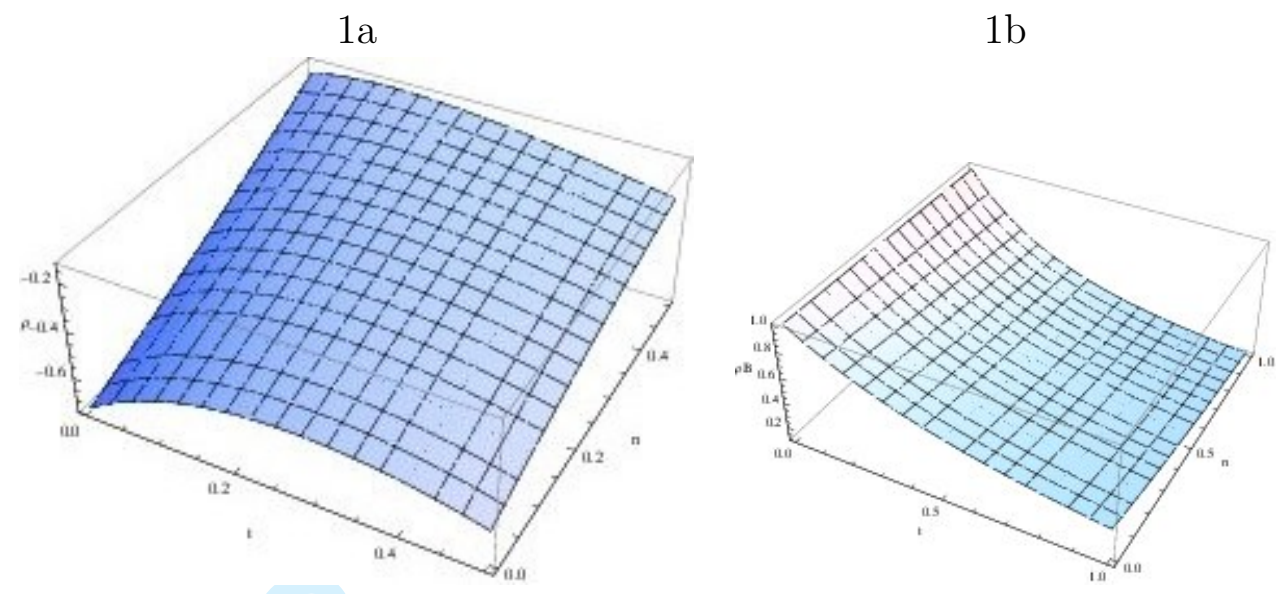

Figure 1: Behavior of $\rho$ and $\rho_{B}$ for Linear Case

the conventional assumption of constant deceleration parameter to explore the exact solutions of field equations. However, for the sake of simplicity, we have used the condition that expansion scaler proportional to shear scalar which gives $b=c^{n}$. We further considered the metric coefficient $c(t)$ in a general form, i.e. $c(t)=(f(t)+\beta)^{k}$, where $f(t)$ is an arbitrary analytic function of $t$. This assumption allows us to consider different forms of $f(t)$ to analyze different solution of field equations. The cosmological parameters are calculated for the given model. Moreover, the isotropy condition $\frac{\sigma^{2}}{\theta} \rightarrow 0$ as $t \rightarrow \infty$ is satisfied for linear and quadratic cases. For exponential case, $\frac{\sigma^{2}}{\theta}$ approaches to a constant value as $t \rightarrow \infty$ and the isotropy condition may be verified for some suitable values of $n$. It is interesting to mention that EoS parameter $\omega$ possesses negative values for all three cases. It can be seen from graphs for a certain value of anisotropic parameter $n, \omega=-1$. This is interesting as it has been proved that the expansion of the universe is accelerating when $\omega \approx-1$ [31]. Moreover, the phantom like dark energy is found to be in the region where $\omega<-1$ and the universe with phantom dark energy ends up with a finite time future singularity known as cosmic doomsday or big rip [32].

We have discussed the exact solutions with only three cases. Many other solutions can be explored by assuming some other forms of $f(t)$. This study will throw some light on the structure formation of the universe, which has astrophysical significance. Similar analysis for other Bianchi type models is 

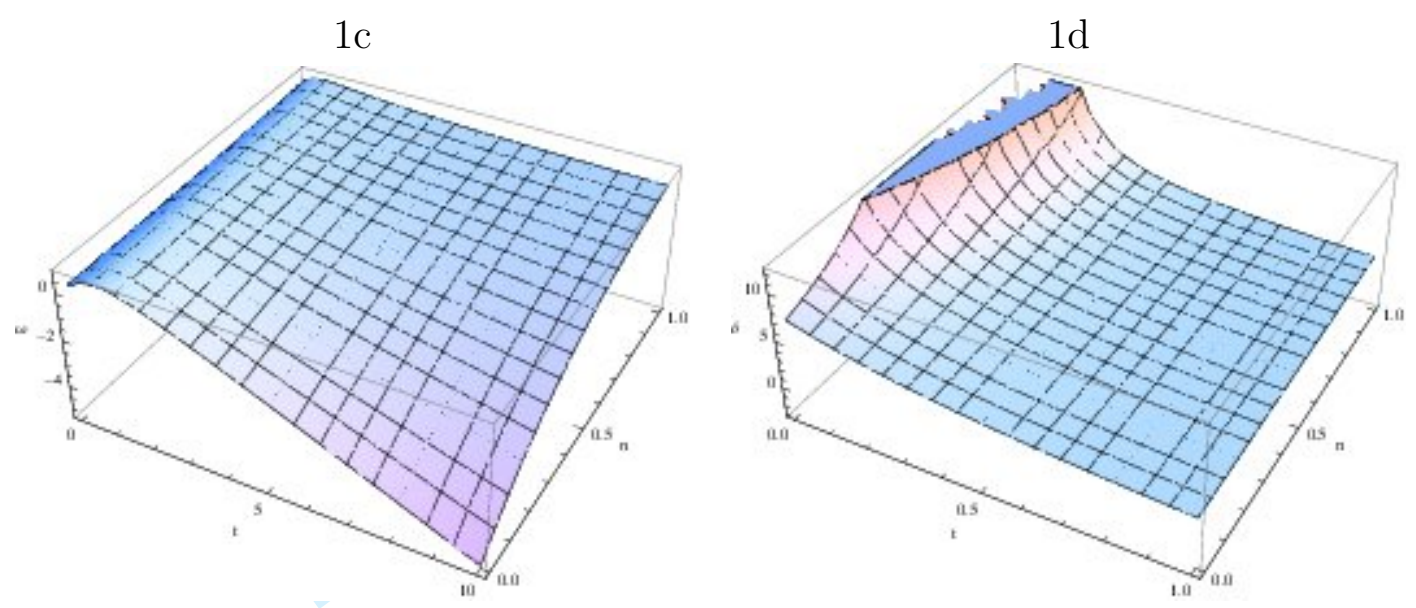

Figure 2: Behavior of $\omega$ and $\delta$ for Linear Case



Figure 3: Behavior of $\gamma$ for Linear Case 

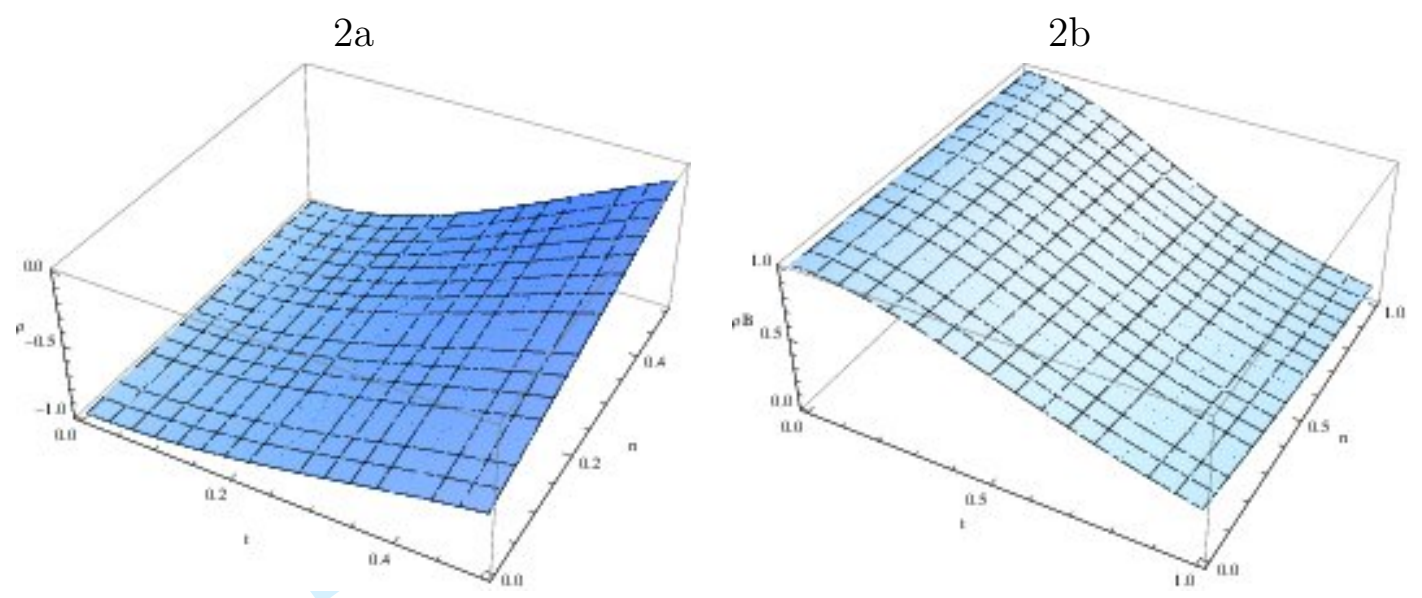

Figure 4: Behavior of $\rho$ and $\rho_{B}$ for Quadratic Case
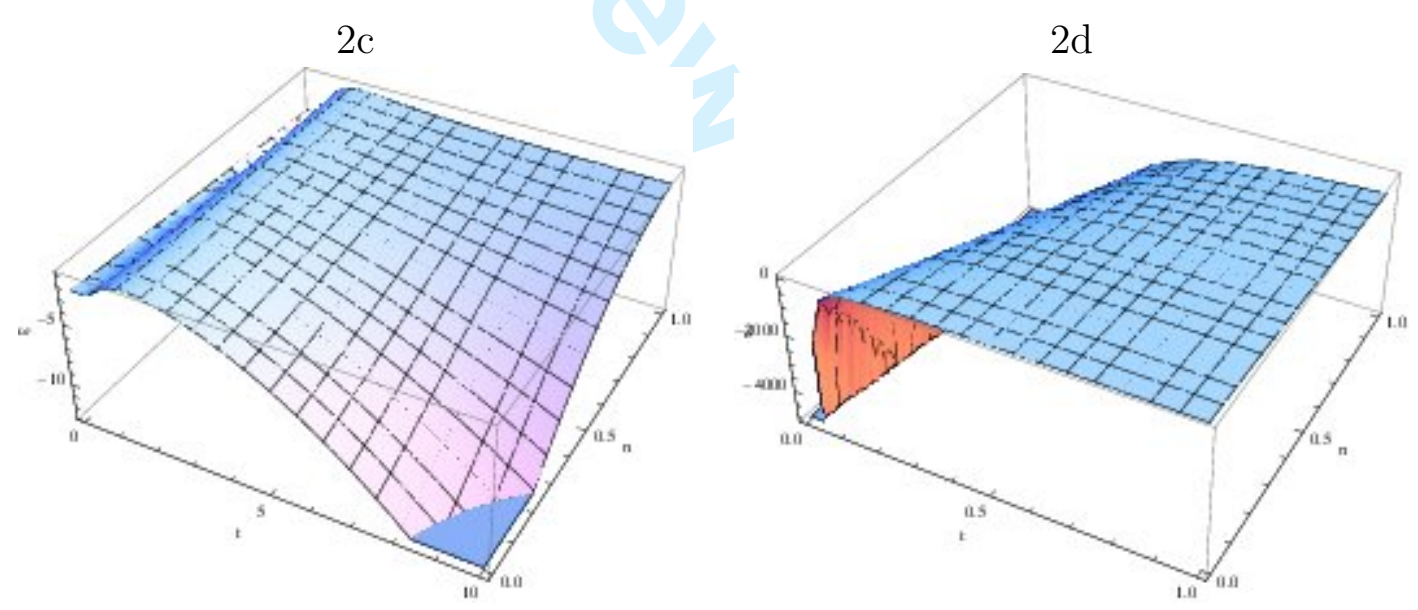

Figure 5: Behavior of $\omega$ and $\delta$ for Quadratic Case 


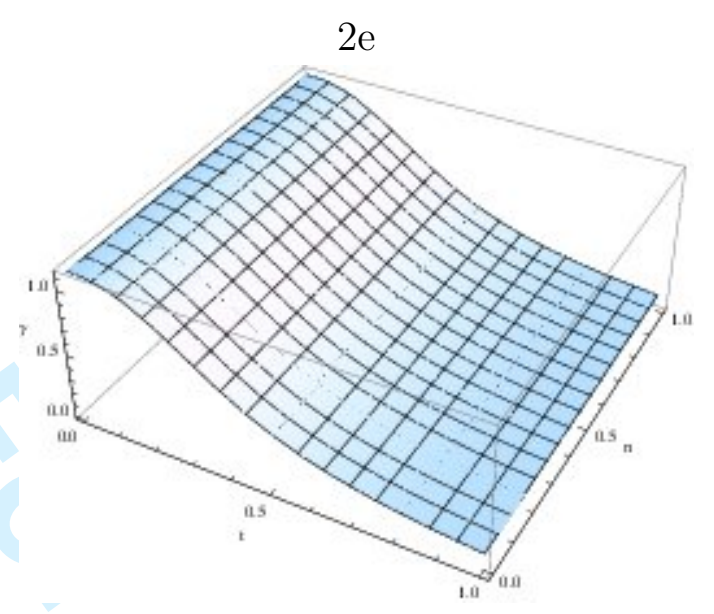

Figure 6: Behavior of $\gamma$ for Quadtratic Case
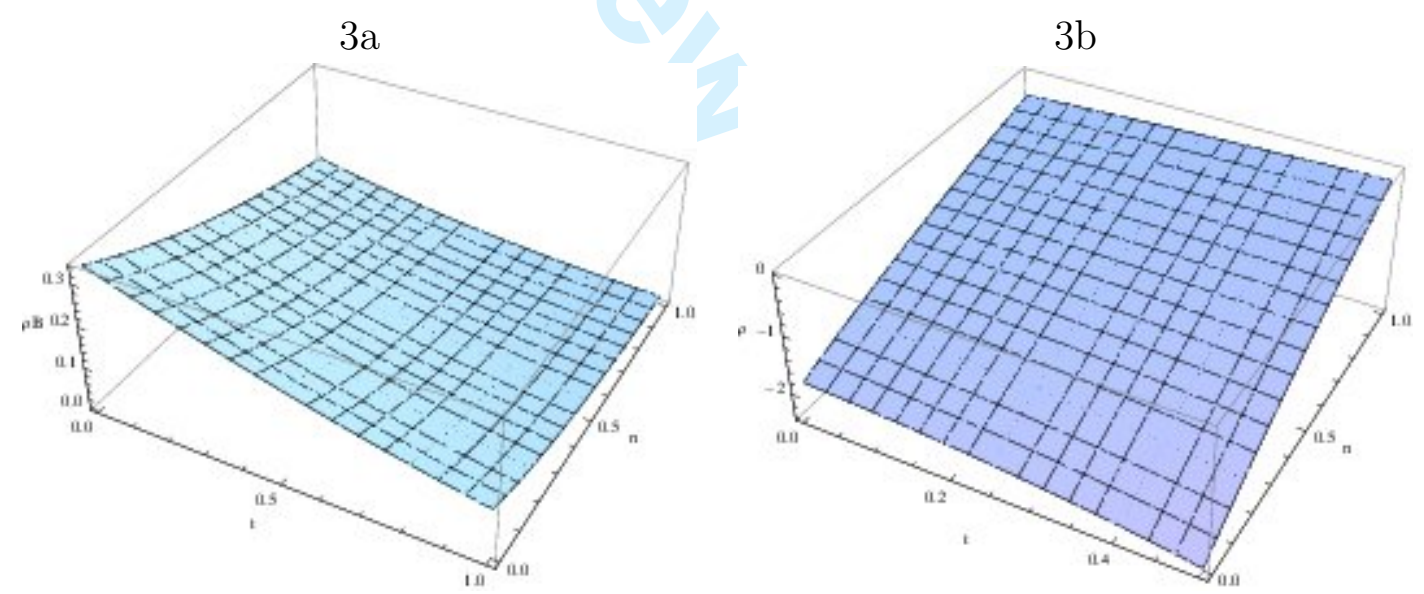

Figure 7: Behavior of $\rho$ and $\rho_{B}$ for Exponential Case 

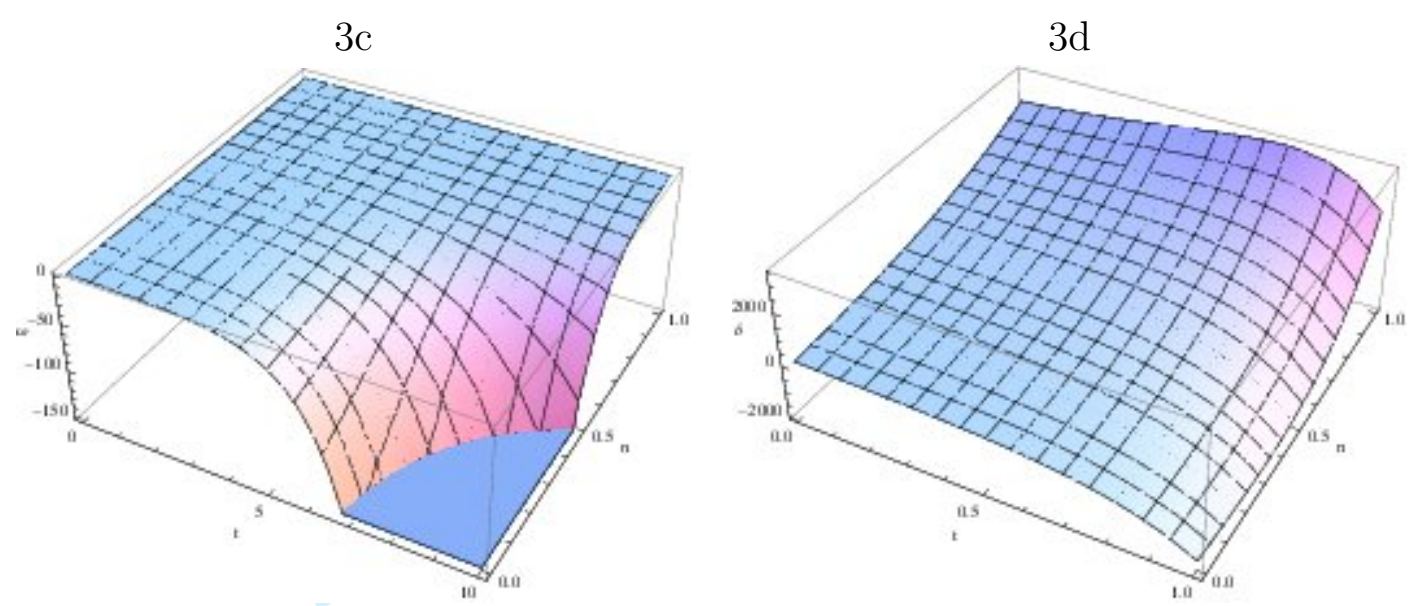

Figure 8: Behavior of $\omega$ and $\delta$ for Exponential Case

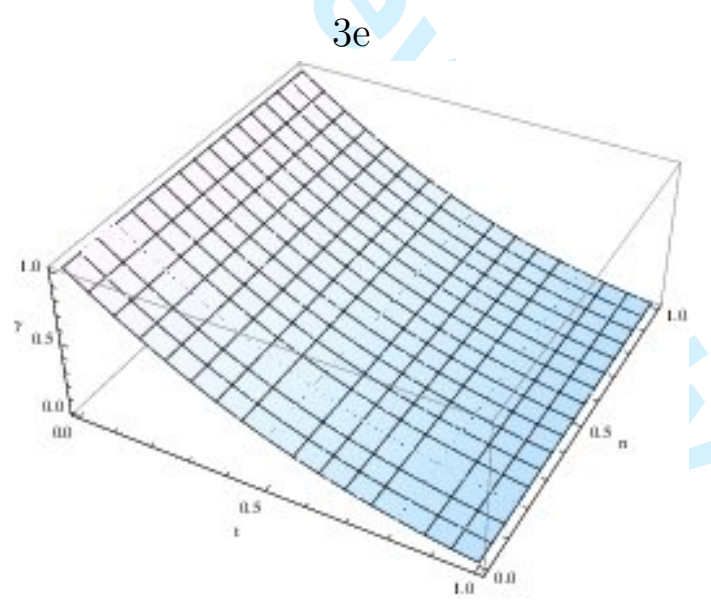

Figure 9: Behavior of $\gamma$ for Exponential Case 
under process.

\section{References}

[1] P.M. Garnavich et al. Astrophys.J. 509(1998)74-79.

[2] S. Perlmutter et al. Astrophys. J. 483(1997)565; S. Perlmutter et al. Nature. 391(1998)51-54; S. Perlmutter et al. Astrophys. J. 517(1999)565586.

[3] A.G. Riess et al. Astron. J. 116(1998)1009-1038.

[4] B.P. Schmidt et al. Astrophys. J. 507(1998)46-63.

[5] R.A. Knop et al. Astrophys. J. 598(2003)102.

[6] M. Tegmark, et al. Astrophys. J. 606(2004)702-740.

[7] P.J.E. Peebles, and R. Bharat. Rev. Mod. Phys. 75(2003)559; P.J.E. Peebles, and R. Bharat. Astrophys. J. L17(1998)325; P.J.E. Peebles, and R. Bharat. Phys. Rev. D37(1998)3406.

[8] T. Padmanabhan. Phys. Rept. 380(2003)235-320.

[9] C. Tortora, E. Piedipalumbo, C. Rubano, and M. Demianski. Astron. Astrophys. 431(2005)27-43.

[10] Cardone, V. F. et al.. Astronomy and Astrophysics. 429(2005)149.

[11] R. Caldwell. Phy. Lett. B545(2002)23-29.

[12] V. Sahni, and A.A. Starobinsky. Int. J. Mod. Phys. D9(2000)4373.

[13] Y. Ma. Nucl. Phys. B804(2008)262-285.

[14] M.S. Berman. Nuovo Cimento. 182(1983)

[15] M.F. Shamir. JETP 150(2016)705.

[16] M.F. Shamir. Astrophys. Space Sci. 361(2016)147.

[17] M.F. Shamir. JETP 150(2016)1127. 
[18] A. Pradhan, H. Amirhashchi, and B. Saha. Int. J. Theor. Phys. 50(2011)2923-2938.

[19] O. Akarsu, and C.B. Kilinc. Gen. Rel. Grav. 42(2010)119-140.

[20] O. Akarsu, and C.B. Kilinc. Gen. Rel. Grav. 42(2010)763.

[21] L. Yadav, and A.K. Yadav. Int. J. Theor. Phys. 50(2011)218-227.

[22] S.D. Tade, and M.M. Sambhe. Int. J. Theor Phys. 51(2012)447454.

[23] R. Jaiswal, A. Pradhan, K. Jotania, and R.K. Khare. Astrophys. Space Sci. 337(2012)401-413.

[24] B. Saha. Int. J. Theor. Phys. 52(2012)1314-1325.

[25] Priyanka, S. Chandel, M.K. Singh, and S. Ram. Global Journal of Science Frontier Research Mathematics and Decision Sciences 12(2012).

[26] B. Saha. Int. J. Theor. Phys.. 52(2012)3646-3657.

[27] M. Sharif, and M.F. Shamir. Class. Quantum Grav. 26(2009)235020

[28] M. Sharif and M.F. Shamir. Gen. Relativ. Gravit. 42(2010)2643.

[29] M. Sharif, and M. Zubair. Astrophys. Space Sci. 330(2010)399.

[30] M. Sharif, and M. Zubair. Int. J. Mod. Phys. D19(2010)1957.

[31] J. Hogan. Nature 448(2007)240.

[32] A.A. Starobinsky. Grav. Cosmol. 6(2000)157; R.R. Caldwell, M. Kamionkowski, and N.N. Weinberg. Phys. Rev. Lett. 91(2003)071301; S. Nojiri, and S.D. Odintsov. Phys. Rev. D70(2004)103522. 